\title{
LES POLITIQUES PUBLIQUES COMPAREES : TOURISME INTELLIGENT OU VRAI PROGRES? LE CAS DES POLITIQUES COMPAREES DE L'ENVIRONNEMENT
}

Peter KNOEPFEL (1)
Corinne LARRUE (1)

Résumé

L'analyse comparée de politiques publiques, qui se distingue de l'analyse comparative des systèmes politiques entiers, a connu un développement rapide dès le debut des années 70 . Ce nouveau type d'analyse reflète le fractionnement actuel des administrations des états industrialisés en crise. Mais cette isolation des politiques publiques analysées et comparées fait courir le danger de perdre une vue

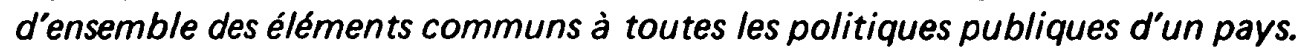

En fait, ce type d'analyse n'a pas encore permis d'elaborer une "theorie comparative des Etats", fondée sur la permanence d'eléments structurels de l'ensemble des politiques publiques des pays (ASHFORD). Mais cet objectif ambitieux n'avait pas été énoncé dès l'origine de cette nouvelle discipline. D'autres espoirs avaient été misés sur les analyses de type "politiques publiques comparées" : initialement, ces analyses devaient permettre de déterminer les conditions exactes du transfert d'éléments et de concepts d'un pays à l'autre, de participer à l'élaboration de stratégies de réforme à l'intérieur des pays étudiés et d'élaborer de nouvelles hypothèses.

On se pose la question de savoir si les recherches de type "politiques publiques comparées" ont pu combler ces espoirs, que la communauté scientifique, les administrations et les financiers avaient portés sur elles. Après avoir dressé un bilan des recherches comparées, menées dans le domaine de la politique de l'environnement, il est montré pourquoi ces trois objectifs se sont avérés im. possibles à atteindre : la rigidité conceptuelle de l'analyse comparée de politiques publiques, sa limitation à un nombre restreint d'eléments composant les politiques étudiées et son incapacité à produire de nouvelles hypothèses sont la cause de l'échec de ce type d'analyse à répondre aux objectifs de départ. Cependant, les trois limites inhérentes à ces recherches constituent en fin de compte un avantage pour ce type d'analyse. En effet, par le biais de la comparaison internationale, on peut mettre en évidence l'existence de facteurs décisifs des politiques publiques étudiées, qui ne peuvent pas étre découverts par le biais de l'analyse non comparative, du fait d'un certain aveuglement culturel inhérent à cette dernière. Les hypothèses développées dans les contextes nationaux sont soumises aux tests empiriques de l'analyse comparée et leur validité est alors remise en cause, ce qui permet d'obtenir un certain niveau de généralisation des hypothèses de départ ; l'analyse comparée met également en évidence des similitudes de modèles explicatifs dans des régions de pays différents ainsi que des variations importantes de ces modèles explicatifs dans différentes régions d'un même pays.

(1) Institut de Hautes Etudes en Administration Publique (IDHEAP) - Lausanne - 
L'analyse de politiques publiques comparées : évolution et raison d'être
L'analyse comparative est à l'heure actuelle scindée en deux courants de pensée :

- Le courant traditionnel dont les représentants, rassemblés autour du journal "Comparative Politics» fondé en 1968, restent préoccupés par la comparaison des éléments de base des systèmes politiques -en témoignent la série "Contemporary Comparative Politics», publiée par J. LA PALOMBA [1] ou encore les recherches menées sur le thème du fédéralisme comparé [2]. Ce type d'analyse repose essentiellement sur la comparaison d'institutions politiques particulières (approches institutionnelles) [3] ou de types de gouvernement (systèmes démocratiques et autres systèmes) [4] ou encore sur la comparaison des systèmes politiques dans leur entier (approche fonctionnaliste de MERTON 1957. ALMOND 1960, et surtout de PARSONS 1960 et MITCHELL 1962. et l'approche systémiste de ALMOND-VERBA ou EASTON 1965).

- Un nouveau courant, né au début des années 1970, s'est orienté vers la comparaison de politiques publiques particulières menées dans le cadre de systèmes politiques différents. La première recherche menée sur une grande échelle qui témoigne de cette évolution est l'étude de HEIDENHEIMER, HECLO et TEICH ADAMS, publiée en 1975, dans laquelle les auteurs comparent les politiques fiscales, les politiques de santé publique, de l'urbanisme et du logemént des Etats-Unis et de certains pays d'Europe (Grande-Bretagne, Suède, Allemagne de l'Ouest et partiellement France et Pays-Bas). C'est cette étude qui a marqué la scission de l'analyse comparative en deux courants.

La nouvelle école s'inspire des analyses de politiques publiques américaines comme celle de LOWI 1972, CAMPBELL 1969, PRESSMAN et WILDAVSKY 1973, ou de BARDACH 1977. L'application de ces conceptions américaines de l'analyse de politiques publiques aux cas européens (Scandinavie, Pays-Bas, Angleterre, Allemagne de l'Ouest) [5] a permis aussi de développer des recherches comparatives dans les pays européens et d'enrichir les concepts analytiques utilisés.

Cette nouvelle école, appelée "Politiques Publiques Comparées» consiste en une analyse des similitudes et des différences de l'impact de politiques menées dans le but de résoudre des problèmes semblables, présents dans les différents pays étudiés. Cette approche utilise un cadre analytique et des indicateurs communs à tous les pays analysés. L'impact de la ou des politiques choisies est évalué et expliqué par les variations des systèmes politico-administratifs (lors de la formation et de la mise en oeuvre de ces politiques), par la position et l'arrangement des acteurs impliqués et le poids plus ou moins dominant de chaque partie prenante. L'analyse de politiques publiques comparées est donc essentiellement une analyse des stratégies politico-administratives permettant de résoudre un problème donné, et ayant recours à différents instruments administra. tifs mettant en jeu des acteurs institutionnels et sociaux. Ces recherches tentent également de prévoir quelles sont les conséquences de la mise en place de stratégies données sous des conditions socio-culturelles et socio-économiques données dans différents pays. 
L'approche qui consiste à distinguer une politique publique en l'isolant de l'ensemble des autres politiques correspond de fait à la situation des Etats "Providence" occidentaux qui subissent une période de crise économique importante : le secteur public en réaction aux contraintes imposées par l'austérité se désintègre, se fragmente au fur et à mesure et se transforme partiellement en institutions corporatistes. La devise semble être "chaque politique pour soi, et le gouvernement pour tous». Les responsables de chaque politique publique, en accord avec les acteurs principaux de leur domaine, essayent d'établir leur propre système de références et de support politique ; la crise de coordination va en s'aggravant et la résistance de ces unités politico-administratives relativement indépendantes, à l'application de programmes gouvernementaux et à la législation parlementaire semble se renforcer. Les gouvernements échouent dans leur lutte contre leurs propres administrations qui préfèrent privilégier leurs relations de clientèle. Cet échec se manifeste par les mauvais résultats obtenus par la mise en oeuvre des politiques publiques, ce qui est le cas de beaucoup de pays industrialisés.

Pour mener ce type d'analyse, orientée vers l'étude de situations concrètes, il semble plus logique de choisir les politiques publiques comme unité d'analyse plutôt que les systèmes politiques entiers. Cela semble être d'autant plus justifié que les problèmes à résoudre, du fait de l'intégration économique internationale, se ressemblent. De plus, certaines politiques publiques se recoupent aussi sur le plan international et ont instauré une communauté politicoadministrative ad hoc, cohérente et résistante aux attaques des acteurs politiques nationaux soi-disant non concernés.

Cette discipline a pris de plus en plus d'importance. Plusieurs domaines ont fait l'objet de recherches comparées. Lors du WZB forum, organisé en 1983 , sur le thème "Cross National Policy Research", plusieurs de ces études furent présentées : celle de VOGEL [6] concernant la politique de l'environnement, celle de LUEBBERT, WILENSKY, HAHN et JAMIESON [7] concernant la politique sociale, celle de HUFNER, MEYER et NAUMANN [8] dans le domaine de l'éducation et celle de STRUMPEL et de SCHOLZ [9] concernant la politique économique. C'est dans ces quatre domaines que semblent se concentrer les investigations des chercheurs à l'heure actuelle; (nous nous sommes fondés sur les recherches présentées à ce forum pour mener notre analyse, car l'ensemble des domaines intéressant l'analyse de politiques publiques comparées y a été présentél.

Mais les résultats de ces recherches mettent en évidence le risque que l'analyse de politiques publiques comparées se détache du contexte culturel et du contexte politique constitués par les autres politiques publiques. L'américain ASHFORD avait souligné ce danger dès 1976, lors d'une conférence à l'Université de Cornell : tout en soutenant l'approche orientée vers les politiques publiques particulières, il avait souligné la nécessité d'une vue d'ensemble : "Perhaps the most interesting thing about policy formation, implementation and evaluation, however one wishes to divide the policy process, is that there are important structural similarities accross all or most policies for any one state. As such differences are understood for a number of states, we may approach something like a comparative policy explanation of 
the state that rivals the one provided by behavioral and economic models of policy" (ASHFORD 1978, p. 13). Et il continue en disant qu'avec la croissance du secteur public "A policy based formulation of state power may be a more accurate representation of how the modern state functions than those built on formulations of electoral, legislative, or party politics) (p.14).

Pour palier ce danger, ASHFORD propose d'utiliser l'analyse des politiques concrètes de différents pays lanalyse menée sur le niveạu régional et même local) pour développer une théorie de l'Etat, fondée sur l'ensemble de ces politiques publiques et non plus sur les éléments traditionnels de légitimité du "politique». Cette proposition tient compte des résultats des analyses de la mise en oeuvre de politiques publiques qui concluent à la séparation de plus en plus évidente entre le complexe politico-administratif et sa légitimité politique. En fait, cette proposition d'ASHFORD permet de concilier la comparaison de politiques publiques, requise par la pratique politique de nos jours, avec la comparaison des systèmes dans leur entier, qui apparaît comme une préoccupation plus théorique.

De plus, l'analyse de politiques publiques comparées, toute fascinante et à première vue pertinente qu'elle soit, si elle utilise un cadre analytique adapté à la complexité des réalités, dépassera les frontières de la politique publique choisie, notamment lors de l'explication de l'impact de cette politique et plus particulièrement lors de l'analyse des variables explicatives de la structure des programmes choisis et des arrangements politico-administratifs (lors de la formation et de la mise en oeuvre du programme). Chaque pays semble avoir son style d'intervention publique, issu d'une tradition politico-administrative, de la constitution générale du système économique et socio-culturel ainsi que de la position de la fonction publique en général. On peut souligner, avec ASHFORD, que les politiques publiques individuelles d'un pays sont plus déterminées par ces variables structurelles que par une volonté explicite du "politique» (gouvernement, parlement, partis politiques, etc.) (ASHFORD 1978, p. 91 et suivantes).

Les preuves empiriques de cette hypothèse ambitieuse sont loin d'être réa. lisées, mais il faut dire qu'elles n'avaient pas été requises au départ. Cependant, on doit se demander quel est l'apport des analyses de politiques publiques de ces dix dernières années lanalyses coûteuses, de longue haleine et bénéficiant de la confiance de l'ensemble de la communauté scientifique), à l'égard des objectifs fixés initialement ; l'analyse comparée de politiques publiques devait au départ, selon les organismes et les personnes qui finançaient ces recherches, pouvoir déterminer les conditions exactes permettant le transfert d'éléments et de concepts d'un pays à l'autre, et participer ainsi à l'élaboration de stratégies de réforme à l'intérieur des pays étudiés ; et enfin, cette analyse devait être capable d'élaborer de nouvelles hypothèses. A-t-elle répondu à ces espoirs ? 
Nous essayerons dans les chapitres suivants d'apporter une réponse à cette question, en nous limitant aux politiques comparées de l'environnement sur lesquelles nous avons nous-mêmes mené une recherche. Nous présenterons un premier bilan de ces recherches sur la base du rapport de David VOGEL pour le WZB forum "Cross National Research» (excellent rapport dont nous partageons dans l'ensemble le point de vue) ; ce premier bilan est assez pauvre eu égard aux objectifs initiaux. Mais, nous essayerons ensuite de développer quelques aspects généraux des limites et de la portée de l'analyse de politiques publiques comparées.

Dans son rapport de décembre 1983, David VOGEL, qui a lui-même mené une recherche comparative de l'intervention publique dans le domaine du contrôle écologique des activités industrielles aux Etats-Unis et en GrandeBretagne, a choisi de présenter une douzaine de projets (parmi les vingt-cinq projets comparatifs connus), qui allaient selon lui au delà de la simple constatation ou description des différences entre les politiques étudiées et qui cherchaient à expliquer ces différences.

Nous mentionnerons certains de ces projets dont la liste se trouve en annexe : le projet de BRICKMAN, JASANOFF et ILGEN analyse les régimes de contrôle de certaines substances chimiques (additifs dans la nourriture, pesticides, produits chimiques utilisés sur les lieux de travail, produits chimiques soumis à déclaration ou autorisation avant la vente) aux Etats-Unis, en France, en GrandeBretagne et en Allemagne de l'Ouest. Cette recherche prend en considération le cadre institutionnel et légal de l'intervention publique, les procédures de contrôle, la formation des décisions et leur mise en oeuvre, et notamment le rôle des tribunaux. Le rôle des experts, des mouvements de protection de l'environnement et des mouvements de consommateurs est particulièrement examiné. Comme les autres analyses de politiques de l'environnement qui comparent les Etats-Unis et des pays Européens (WILDAVSKY 1983, KELMAN 1981, LUNDOVIST 1974/1980 et VOGEL 1983), cette recherche aboutit à la constatation que la politique américaine est beaucoup plus systématique et que les politiques européennes sont plus souples. Cette distinction principale s'explique par une distribution des pouvoirs différente dans les deux systèmes politiques: le fractionnement du pouvoir, qui permet une influence politique de plusieurs acteurs institutionnels et sociaux, mène outre Atlantique à une formalisation plus importante, à une intervention judiciaire plus fréquente et à un taux de conflit considérable. Les systèmes européens présentent par contre un modèle commun de coopération entre les acteurs concernés, une tradition de fréquentes consultations et un taux d'intervention judiciaire beaucoup moins important ; le processus de décision est par conséquent moins conflictuel. Malgré ces différences dans les procédures d'élaboration des programmes, ces programmes eux-mêmes, et notamment les normes à imposer, sont relativement proches. Les auteurs expliquent ce phénomène par la présence d'organisations internationales pour la protection des intérêts de l'industrie chimique et par l'échange des résultats scientifiques, de plus en plus courant de la part de la communauté scientifique et des mouvements écologiques. 
L'étude de KELMAN 1981 présente quant à elle, une comparaison des politiques de santé publique et de sécurité du travail en Suède (pays connu pour son gouvernement social-démocrate traditionnellement au pouvoir) et aux EtatsUnis. Cette recherche a abouti à des résultats similaires : dans les deux pays, les autorités publiques compétentes ont choisi une marge de sécurité importante, mais les décisions des autorités suédoises ont été bien mieux acceptées par les industries concernées qu'aux Etats-Unis ; les administrations américaines se sont vues imposer une règlementation détaillée par le législateur, notamment pour les procédures à suivre, tandis que les administrations suédoises ont eu plus de liberté pour choisir entre différentes procédures. De même, les autorités suédoises ont choisi une approche plus souple pour le contrôle et l'application des sanctions à l'égard des entreprises. Ainsi, l'auteur constate que les tribunaux américains jouent un rôle remarquable tant dans les procédures de formulation des programmes que pour leur mise en oeuvre, alors que le pouvoir judiciaire est quasiment absent en Suède. Le système américain se montre ici aussi plus conflictuel, plus rigide vis-à-vis des entreprises et plus interventionniste que le système européen étudié. Contrairement à ce que l'auteur attendait, le système social démocrate, essentiellement voué, de par son idéologie, à la protection des ouvriers, se montre plus flexible, moins conflictuel et plus consensuel dans ses relations avec les entreprises. KELMAN conclut que les normes sont aussi rigides dans les deux pays ; il explique cette similitude par des facteurs inhérents aux politiques étudiées (éducation et formation comparables des fonctionnaires, etc.), plutôt que par des caractéristiques plus générales de ces deux pays.

Une autre recherche comparative a été menée par le centre IIASA (KUNREU. THER et LINNEROOTH, 1982) en RFA, en Grande-Bretagne, aux Pays-Bas et aux Etats-Unis sur les politiques d'implantation de grandes entreprises, qui ont des impacts sur l'environnement sur une grande échelle. Cette étude analyse l'influence de l'organisation de l'administration et des procédures suivies, dans la définition de l'intérêt public lors de la décision d'implantation de ces entreprises. Ainsi il apparaît que la décision finale d'implantation est influencée par le mo. ment où l'intervention de la population locale dans le processus est possible (cette intervention se fait plus tôt en Californie que dans les cas Européens étudiés), par l'existence d'une (cas des Etats-Unis) ou de plusieurs administrations publiques aux différents échelons de l'Etat (cas de la RFA) et par la distribution du pouvoir de décision entre l'Etat central et les collectivités locales.

Dans une autre étude, LUNDQVIST explique les différences entre les politiques de lutte contre la pollution atmosphérique en Suède et aux Etats-Unis (la politique américaine étant plus institutionnalisée, plus rapide et moins consensuelle que la politique suédoise) par des caractéristiques particulières à chacun des deux pays, plutôt que par des approches techniques différentes : le député américain qui est fortement impliqué dans la formation des programmes de la politique de lutte contre la pollution atmosphérique est considéré comme responsable du programme mais pas de sa mise en oeuvre. En revanche, 
son homologue suédois, appartenant à la majorité, partage la responsabilité du succès de cette politique avec son gouvernement. De plus, le système unitaire de gouvernement en Suède autorise un contrôle intégral de la politique publique, tandis que le système fédéral américain permet aux Etats membres de participer a la mise en oeuvre de programmes relativement stricts, ce qui constitue un frein potentiel à la rigidité de ces programmes. Cette organisation, issue de la constitution, est un élément structurel des processus de mise en oeuvre aux Etats-Unis. Mais, malgré des différences importantes entre les programmes des deux pays, LUNDQVIST conclut à la similitude à long terme de l'ensemble des impacts des deux politiques étudiées.

Les résultats de la recherche de VOGEL (1983), qui compare les politiques de I'environnement des Etats-Unis et de la Grande-Bretagne sous I'angle spécifique du contrôle des activités industrielles, sont similaires. Cette recherche confirme le caractère conflictuel et légaliste de l'approche américaine qui contraste avec le style coopératif, plus informel et plus ouvert aux négociations avec les entreprises, de la Grande-Bretagne. Pour expliquer ces différences, VOGEL va plus loin que dans les recherches précédentes : pour lui, la clef de l'explication ne se trouve pas au niveau des systèmes constitutionnels, car ils sont très proches sur le plan de l'intervention publique dans le secteur industriel (ces deux pays partagent à peu près la même philosophie libérale), ni au niveau de la politique de l'environnement, car le même style d'intervention publique se retrouve dans les deux pays pour d'autres politiques publiques. L'explication des différences se trouve plutôt dans la tradition de relations entre gouvernement et industries, différentes dans les deux pays : l'industrie britannique est plus disposée à accepter un certain niveau de contrôle public, les fonctionnaires sont beaucoup plus qualifiés et respectés au Royaume-Uni et le public anglais est plus disposé à accepter une certaine coopération entre industries et gouvernement.

Le rapport VOGEL présente finalement un projet de recherche, qui compare les politiques publiques de lutte contre la pollution atmosphérique dans sept pays européens, qui a été mené par un groupe relativement nombreux de chercheurs sous la direction de l'Institut International pour l'Environnement et la Société à Berlin (KNOEPFEL, WEIDNER, 1982/1983), (cette recherche est présentée sur la base de résultats intermédiaires, le rapport final n'étant pas disponible à l'époque). Cette étude, dont le cadre analytique est censé être l'un des plus exhaustifs, analyse les enjeux politiques concernant la formation et la mise en oeuvre des programmes de lutte contre la pollution atmosphérique par le dioxyde de soufre. Elle montre que, dans la plupart des pays étudiés, les difficultés de la mise en oeuvre peuvent être attribuées en partie à la déficience des programmes eux-mêmes et que, quoiqu'en disent les pouvoirs publics, il est quasiment impossible de mesurer réellement les impacts des politiques publiques menées sur le plan local et d'expliquer les tendances observées de la pollution seulement par l'activité de l'administration de l'environnement régionale ou locale. Cette recherche met plutôt en évidence des relations entre développement économique, politique énergétique et qualité de l'environnement. Le rapport 
final de cette étude, qui n'est pas pris en compte dans le rapport VOGEL, montre cependant des différences d'impact de ces politiques dans les 14 régions incluses et notamment pour les 30 localités étudiées plus précisément. Les différentes combinaisons de facteurs qui expliquent la répartition de l'intervention régulatrice de l'administration dans les politiques de mise en oeuvre des régions étudiées sont mises en évidence. Les différences observées entre les régions des différents pays sont expliquées globalement par les caractères propres de cette politique de lutte contre la pollution atmosphérique qui se réalise essentielle. ment sur le niveau régional (rapports de force entre les acteurs, position, capacité et composition de l'acteur institutionnel principal, structure industrielle et situation économique). Les similitudes observées entre les régions d'un même pays sont expliquées par les caractéristiques propres à ce pays, qui se retrouvent également pour d'autres politiques publiques. Ainsi, les dimensions explicatives se situent dans un premier temps, pour les différences observées, à l'intérieur des politiques étudiées et dans un deuxième temps, pour les caractéristiques communes, dans la structure générale de l'ensemble des politiques publiques des pays étudiés.

\section{Le bilan de ces quelques recherches}

Nous pouvons retenir deux éléments importants de l'état actuel de l'analyse comparée dans le domaine de l'environnement, telle qu'elle est présentée dans le rapport VOGEL :

- Les recherches concernant le contrôle des produits chimiques (BRICKMAN, JASANOFF et ILGEN, 1982), la politique de santé publique et de sécurité du travail (KELMAN, 1981) et les recherches plus orientées vers les processus de mise en oeuvre (implantation de grandes entreprises et $\mathrm{SO}_{2}$ ) se sont avérées partiellement capables de distinguer, parmi les facteurs explicatifs, ceux qui relèvent des caractéristiques propres aux politiques étudiées et ceux qui relèvent de l'ensemble des politiques publiques des pays. Cette dissociation, quoique partielle, permet de mettre en évidence les éléments décisifs des politiques étudiées, ce qui permet d'élaborer des stratégies de réforme; on peut envisager un transfert de connaissances, d'expériences et même d'éléments de program. mation et de mise en oeuvre d'un pays à l'autre, pour tous les facteurs qui ne dépendent pas des structures fondamentales communes à l'ensemble des politiques publiques d'un pays. Les autres transferts risquent d'avoir des impacts imprévisibles et perturbants. Mais nous sommes loin de l'espoir des premières heures de l'analyse de politiques publiques comparées dans le domaine de l'environnement : la ligne de démarcation entre politique publique particulière et éléments constitutifs de l'ensemble des politiques publiques d'un pays n'est pas suffisamment tracée pour pouvoir en tirer des conséquences. Le débat sur les transferts internationaux reste ouvert. VOGEL tire des conclusions similaires (p. 73 et suivantes) ; selon lui, la définition de cette ligne de démarcation dépend de la profondeur des recherches empiriques entreprises (le droit comparé, par exemple, proposera plus fréquemment des transferts que les recherches 
plus approfondies de mise en oeuvre) et de l'intérêt du chercheur. L'auteur constate que les recherches présentées se distinguent essentiellement par leur définition de la variable dépendante : si on cherche à expliquer les différences conceptuelles des politiques publiques dans leur entier, on va considérer les politiques choisies comme exemplaires, c'est-à-dire comme représentatives d'un groupe de pays (par exemple : «industrial regulation», cf. VOGEL, 1983). A cette orientation correspond un choix particulier de pays : on choisira des pays dont on sait par avance que le style d'intervention publique est différent ; dans ce cas, on hésitera à formuler des propositions de transferts d'un pays à l'autre. Par contre, si on s'intéresse aux différences d'impact de politiques publiques conçues de façon semblable, on cherchera plutôt à déterminer les éléments caractérisant la politique elle-même ; dans ce cas, on sera beaucoup plus porté à formuler des propositions de transferts. Ces deux approches ne sont toujours pas conciliables, ni conciliées aujourd'hui et elles peuvent mener à des conclusions contradictoires quant à la question des transferts internationaux. Cette question, soulevée principalement par les administrations nationales et internationales concernées par ces politiques, qui était à l'origine de ces recherches entreprises, n'a toujours pas trouvé de réponse ; dans ce sens, le bilan de ces analyses est plutôt maigre.

- Le second élément que nous pouvons retenir de ce bilan concerne l'hypothèse énoncée de la similitude des impacts des politiques publiques de l'environnement des pays occidentaux industrialisés, quels que soient les stratégies, les programmes administratifs étudiés, les structures de mise en oeuvre ou les caractéristiques fondamentales des politiques publiques des pays ( «convergence hypothesisy). VOGEL, BRICKMAN et al., KELMAN et LUNDQVIST font remarquer que "la mise en oeuvre des politiques semble être plus uniforme que leur élaboration. Bien que les différents pays aient répondu de façon très différente à la pression publique pour améliorer la qualité de l'environnement dans les années 60 et au début des années 70 , en fin de compte, les politiques publiques, coincées d'un côté par les contraintes économiques et de l'autre par des problèmes sérieux de santé publique, ont convergédans le même sens", (VOGEL, p. 18). Les auteurs mentionnés avancent I'hypothèse d'une telle convergence pour le long terme, pour l'ensemble des territoires nationaux (du point de vue de la mise en oeuvre) et pour les impacts immédiats (et non pour les résultats réels ou politiques). Est-ce le signe d'une renaissance du fonctionnalisme Parsonien, fondé sur l'équilibre des systèmes et de leurs fonctions ? Et dans ce cas, cette hypothèse est beaucoup trop abstraite pour se plier à la recherche empirique : ou alors est-ce que le niveau de pression des problèmes (équivalent dans les pays étudiés) est la seule explication des interventions politicoadministratives ? La plupart des recherches comparatives menées jusqu'à nos jours ne semblent pas capables de répondre de façon satisfaisante à cette question, bien que ce soit justement par l'analyse comparée que l'on ait voulu répondre à cette question théorique. Le bilan des recherches de politiques publiques comparées apparaît faible sur ce point également. 
La portée limitée de de l'analyse des politiques publiques
Nous tenterons de montrer, au cours des prochains chapitres, que ce jugement est finalement trop sévère et nous montrerons pourquoi les espoirs initiaux étaient en fait irréalisables.

Comme nous l'avons vu, les analyses de politiques publiques comparées ne permettent pas (ou en partie seulement) de distinguer, parmi les facteurs explicatifs, ceux qui relèvent de l'ensemble des politiques publiques des pays. Elles ne permettent pas non plus de prévoir exactement les conséquences d'un transfert d'éléments de programme ou de mise en oeuvre d'un pays à un autre (même dans le cadre d'un contrôle très sévère). Cette incapacité est en fait inhérente à toute comparaison internationale : elle provient de la nécessaire sélectivité des concepts retenus, de la non-prise en compte des effets cumulatifs de l'impact de l'ensemble du débat politique (qui apparaissent souvent distincts des éléments isolés retenus) et de la difficulté de construire de nouvelles hypothèses théoriques.

Pour permettre la comparaison des données recueillies dans des pays différents, toute recherche comparative qui veut aller au delà d'une simple juxtaposition des rapports nationaux (telle qu'on la pratique souvent dans le cadre des organisations internationales) doit déterminer précisément les variables "dépendantes" à expliquer et retenir un nombre nécessairement limité de variables "explicatives». Il faut également déterminer un certain nombre d'indicateurs communs à tous les pays, qui caractérisent ces deux types de variable ; cela devient encore plus nécessaire lorsque les recherches dans les pays étudiés sont menées par des personnes différentes.

Le concept analytique qui détermine ces dimensions et ces indicateurs est choisi de façon discriminante et préalable : discriminante, car on ne va inclure que les dimensions explicatives qui sont apparues fondamentales lors d'études expérimentales dans un ou plusieurs pays ; préalable, car au début de la recherche on ne sait pas si dans tel ou tel pays choisi on retrouvera réellement des caractéristiques communes et distinctes qui ont été à l'origine du choix de ces pays. La constatation de ces similitudes ou de ces différences est la première étape d'une recherche comparative ; l'anticipation de cette étape, sur la base de recherches préliminaires, qui influence le choix des pays et des régions étudiées, peut s'avérer inadaptée pour répondre à la question posée. Et il est souvent trop tard, une fois la première étape réalisée et les premières constatations faites, d'arrêter la recherche en cours, vu les investissements déjà réalisés. De plus, toute conceptualisation nécessite l'élaboration d'une typologie qui permet de mettre en relation les caractéristiques des variables explicatives avec les observations que l'on veut expliquer. La construction de cette typologie risque d'être biaisée et de ne permettre aucune analyse en dehors du pays ou du continent pour lequel elle a été instituée. L'échec des modèles fonctionnalistes américains des années 60 , dû à leur américano-centrisme, montre bien cette limite fondamentale de l'approche comparative. 
Contrairement aux espoirs énoncés initialement, les politiques publiques comparées n'ont pas permis de contribuer directement au débat politique intérieur. Cela provient essentiellement de la sélectivité conceptuelle de ce type d'analyse. Ce n'est pas toujours le refus de résultats inopportuns ou indésirables qui amène les politiciens locaux, régionaux ou nationaux à critiquer les recommandations issues de l'analyse comparée ; ces critiques sont parfois fondées, car les recherches n'ont pas pu tenir compte de l'ensemble du débat politique né autour d'un cas particulier que l'on a étudié même d'une façon précise. Car ce cas particulier comme les éléments plus généraux constituant une politique publique ont été pris en considération dans la recherche pour d'autres motifs que celui d'une stratégie de réforme de ce cas particulier. Ainsi, pour formuler des recommandations immédiates orientées vers la pratique politique d'un pays étudié, il faut des recherches complémentaires dans ce pays, ou alors organiser la recherche comparative avec des "teams») de recherche nationaux.

La troisième limitation de l'analyse comparée est son incapacité à construire de nouvelles hypothèses plus élaborées que les hypothèses développées dans les contextes nationaux. La méthode comparative s'avère aussi faible de ce point de vue qu'elle apparaît forte pour tester les hypothèses initiales. Cette dernière capacité, déjà indiscutable sur le plan méthodologique (augmentation du nombre des unités d'analyse avec des caractéristiques différentes) est largement reconnue, ce qui rend le comparatiste peu perceptible à l'incapacité de l'analyse comparée à créer de nouvelles hypothèses. Cette limite, ici encore en opposition avec les espoirs initiaux, est donc moins évidente, mais elle est plus sérieuse. La prise en compte des expériences réalisées dans les autres pays est fructueuse et innovatrice, mais elle reste éclectique et le contrôle de processus entiers s'avère difficile voire même impossible à réaliser dans le cadre de l'analyse de politiques publiques comparées stricto sensu. Par manque de conditions "ceteris paribus». la comparaison internationale aura toujours des difficultés à établir des conditions expérimentales (CAMPBELL 1969), qui sont pourtant à notre avis nécessaires pour l'élaboration de nouvelles hypothèses. Cependant, cette limite pourrait être en partie surmontée, en combinant les comparaisons diachroniques et synchroniques, c'est-à-dire en allongeant les périodes pendant lesquelles les politiques publiques sont étudiées. De même, la combinaison de la comparaison internationale et de la comparaison interrégionale ou intercommunale permettrait d'échapper en partie à ce problème.

Aussi paradoxal que cela puisse être, la rigidité conceptuelle et méthodologique inhérente à toute analyse de politiques publiques comparées, qui crée ces limitations, est à l'origine des avantages de ce type d'analyse. Ces avantages, différents de ceux que l'on pouvait attendre, sont décrits dans le chapitre suivant. Ils rendent ce bilan des recherches comparées moins maigre qu'il ne paraît. 
Les avantages de l'analyse de politiques publiques comparées
La distinction entre les facteurs politico-administratifs explicatifs propres à une politique publique particulière et les facteurs communs à l'ensemble des politiques publiques d'un pays est difficile à réaliser du fait de la sélectivité et de la rigidité de l'approche comparative ; mais cette même sélectivité permet également de découvrir un certain nombre de facteurs explicatifs, qu'ils soient propres à une politique particulière ou communs à l'ensemble des politiques, que l'on ne saurait découvrir par une simple analyse limitée à un seul pays. Cette découverte est rendue possible par l'application systématique d'un cadre ana. lytique commun dans les différents pays et par le transfert d'un pays à l'autre des hypothèses et de la typologie des variables étudiées. Cette stratégie de recherche de la comparaison internationale de politiques publiques fait souvent ressortir des éléments explicatifs de certains impacts que l'on ne peut percevoir dans son propre pays qu'au travers de ces lunettes comparatives. Les concepts analytiques comparatifs permettent de mettre en évidence des liens entre certaines structures et les politiques publiques étudiées, qui étaient ignorés auparavant. Cette découverte reste bien sûr occasionnelle et dépend du choix des variables étudiées. Mais il faut remarquer que c'est cette même sélectivité des concepts analytiques qui permet de faire ressortir de telles variables du contexte des politiques étudiées. C'est grâce à l'approche comparative que l'on peut vaincre l'aveuglement culturel qui cache le rôle parfois décisif de facteurs socio ou politico-administratifs dans la réalisation de certaines politiques publiques. Des stratégies de réforme peuvent être alors élaborées sur la base des mécanismes de coercition et de soutien que l'analyse comparée aura fait découvrir.

Ainsi, l'analyse de politiques publiques comparées permet d'approfondir les connaissances que le chercheur peut avoir de son propre pays (VOGEL, 1983, p. 84 ; KNOEPFEL et WEIDNER, 1983, p. 192).

Un autre avantage de l'analyse de politiques publiques comparées, lié lui aussi à une limite fondamentale de ce type d'analyse (sa faiblesse théorique), réside dans sa capacité à réfuter (falsify) les hypothèses issues des seuls contextes nationaux. L'analyse comparative n'est pas capable, en principe, de créer de nouvelles hypothèses, mais elle peut tester empiriquement un ensemble d'hypothèses qualifiant l'influence de telle ou telle variable sur les décisions politiques ou sur leurs impacts. Par le biais de la comparaison internationale, le chercheur découvre des éléments limitant la généralisation des hypothèses initiales, ce qui lui permet d'introduire de nouvelles conditions à leur validité. La recherche $\mathrm{SO}_{2}$ a par exemple montré que l'hypothèse d'une meilleure prise en compte de la qualité de l'environnement par les administrations, du fait de l'ouverture au public des procédures d'autorisation administrative, n'était pas vérifiée; cette hypothèse a été développée aux Etats-Unis et en Europe Centrale (Allemagne de l'Ouest) ; la comparaison internationale a pu mettre en évidence que la qualité écologique de l'action administrative peut être aussi élevée dans des systèmes relativement fermés, tels que le système anglais ou italien, que dans des systèmes plus ouverts. Cet apport de l'analyse comparative pour la généralisation d'un certain nombre d'hypothèses est selon VOGEL encore limité 
Les politiques publiques comparées : tourisme intelligent ou vrai progrès ? 57

Le cas des politiques comparées de l'environnement

a quelques exemples trop peu nombreux (p. 91 et suivantes). Cette approche devrait être développée dans l'avenir. II faut souligner que c'est souvent une tåche peu satisfaisante, voire même gênante, pour le chercheur de relativiser les résultats de ses collègues; la recherche comparative fait courir le risque aux chercheurs d'être de plus en plus isolés dans leur propre pays.

Un autre avantage de l'analyse comparée concerne l'hypothèse énonçant la convergence des impacts des politiques publiques qui tendent à résoudre le même problème, quelles que soient les différences des programmes. Cette hypothèse intéressante a été énoncée dans le cas d'impacts à long terme et dans le cadre de l'ensemble du territoire d'un pays. Mais, même pourvue de cette réserve fondamentale, cette hypothèse n'est pas assez précise ; elle ne peut être testée empiriquement car le nombre des observations est trop peu important (nombre d'observations $=$ nombre de pays) face aux très nombreux facteurs explicatifs qui s'excluent les uns des autres (PRZEWORSKI, 1983, p. 13). Ce dilemme existe pour toutes les recherches comparatives qui retiennent les pays entiers comme unité d'analyse. La prise en compte d'unités d'analyse régionales et locales permettrait d'augmenter le nombre d'observations de la variable «dépendante» et mènerait à des résultats plus précis.

Outre ces arguments méthodologiques, on peut avancer un argument plus substantiel en faveur du choix d'unités d'analyse régionales ou locales. Cet argument vaut essentiellement pour le domaine de la politique de l'environnement : l'état de l'environnement (degré de pollution) ainsi que l'exposition de la population à cette pollution ne peuvent être observés au travers de données agrégées sur l'ensemble du territoire national. On ne peut donc pas théoriquement apporter une réponse valable à la question aussi vieille que controversée : "Do Policies matter ?" dans le domaine de l'environnement, si l'on ne tient pas compte du niveau local et régional. De plus, selon nous, une analyse de politiques publiques comparées doit toujours comprendre l'analyse des processus de mise en oeuvre qui n'ont qu'exceptionnellement lieu sur le plan national. Les analyses nationales de mise en oeuvre de politiques publiques de plus en plus nombreuses nous ont appris que l'impact de la mise en oeuvre d'une politique variait considérablement selon les régions, en fonction de la structure du problème, de l'arrangement des acteurs politico-administratifs, mais également en fonction des relations de pouvoir existant sur le niveau local et régional. Suivons donc les recommandations que nous pouvons tirer de ces recherches nationales de la mise en oeuvre de politiques en prenant comme unité d'analyse des unités de mise en oeuvre réelles (régions et/ou collectivités locales) (ASHFORD, 1978, p. 87 et suivantes ainsi que la littérature mentionnée ci-dessus). A l'heure actuelle il n'existe que deux analyses de politiques publiques comparées dans le domaine de l'environnement qui aient réellement suivi ce conseil (le projet $\mathrm{SO}_{2}$ de IIES ou le projet "The Siting of LEG Facilities in Four Countries" de IIASA). Mais seul le projet $\mathrm{SO}_{2}$ a pris en compte plusieurs collectivités locales (au total 30 ) dans plusieurs régions (14) et dans plusieurs pays (5). Les résultats de cette recherche mettent en évidence des différences intéressantes du point de vue des politiques régionales de mise en oeuvre. 
C'est au cours de cette recherche $\mathrm{SO}_{2}$ que nous avons appris à poser la question "Do policies matter ?» d'une autre façon. Nous avons pu montrer que l'intervention publique à l'intérieur d'un même pays et même d'une même région peut considérablement varier du point de vue de son intensité, de sa qualité et de ses impacts. Cela montre bien que la question intéressante réside dans l'explication des modèles de discrimination qui privilégient des collectivités locales et des régions par rapport à d'autres. La question «Do policies matter ?» est posée d'une façon trop générale pour le domaine de l'environnement. Ce qui compte, c'est surtout «For whom do policies matter ?». En posant la question de cette façon, on peut apporter des réponses plus précises, plus proches des réalités politiques (malgré la réserve énoncée au chapitre précédent) et caractérisant autant les politiques publiques particulières que le style général d'intervention publique d'un pays. Mais on peut se demander en quoi l'approche comparative est plus capable de découvrir et d'expliquer de tels modèles de discrimination qu'une approche limitée à un seul pays. Quel est l'apport supplémentaire d'une recherche comparative?

La recherche $\mathrm{SO}_{2}$ nous a montré que l'hypothèse de la portée limitée des programmes nationaux suggérée par des recherches non comparatives est valable pour tous les pays européens. Les modèles de discrimination observés dans les régions s'expliquent souvent par des facteurs autres que les programmes nationaux. Ces facteurs sont constitués essentiellement par la structure et la pression des problèmes, et par la perception que les acteurs concernés ont de ces problèmes. De plus, nous avons constaté que dans tous les pays étudiés, l'inter. vention publique dans les différents espaces, à l'intérieur des régions, n'est expliquée qu'en partie seulement par la pression réelle du problème (concentration de $\mathrm{SO}_{2}$ dans les différents espaces). L'explication réside également, en partie, dans des modèles de distribution "politique» qui proviennent des processus d'interaction des acteurs concernés, de la capacité politique et technique des acteurs sociaux, des caractéristiques de l'administration régionale de contrôle (composition, dotation en personnel, capacité d'analyse) et des priorités réelles ou intentionnelles de la politique de mise en oeuvre de cette administration.

La définition de ces dimensions explicatives de la politique régionale de mise en oeuvre et la détermination de leur influence se trouvent mieux expliquées dans le cadre de la comparaison internationale : les similitudes des modèles explicatifs observées dans des régions de différents pays sont remarquables. De ce fait, le chercheur peut constater une convergence non plus des impacts mais des modèles explicatifs de la différence de ces impacts. C'est la découverte de ces modèles explicatifs des impacts des politiques publiques à l'intérieur des pays qui constitue le réel apport des politiques publiques comparées. Les faits observés dans différents pays peuvent être semblables, voire identiques. Ce n'est pas la comparaison des faits, mais la comparaison de leurs modèles explicatifs qui constitue l'apport fondamental de l'analyse de politiques comparées. 


\section{NOTES}

1. La collection "Contemporary Comparative Politics Series» comprend des analyses comparatives des domaines suivants : Législation (J. BLONDEL), Cadres culturels des législations (H.W. EHRMANN), les limites du gouvernement (C.J. FRIEDRICH), Les élites politiques (R.D. PUTNAM), La corruption (J.C. SCOTT), Les idéologies (P. GOUREVITCH), Les systèmes électoraux (D.W. RAE et M. TAYLOR).

Ces ouvrages ont été édités par Prentice-Hall, Inc., Englewood Cliffs, N.J., à partir de 1972.

2. Voir par exemple, BARTHALAYB. : Le Fédéralisme, Presses Universitaires de France, (Que Sais-Je ? $N^{\circ}$ 1953), Paris, 1981. Ou DUCHACEK I.D. : Comparative Federalism - The Territorial Dimension of Politics, HOLT/ RINEHART/ WINSTON, New York, 1970. ELAZAR D.J. : Federalism and Political Integration, TURTLEDOVE, RAMAT GAN 1979. FRIEDRICH C.J. : Tendances du Fédéralisme en Théorie et en Pratique, Bruxelles, Institut Belge de Science Politique, 1971. MERKL P.H. : Modern Comparative Politics, HOLT/ RINEHART/WINSTON, New York 1970, pp. 232-261. ROK$K A N S$., URWIN D.W. : The Politics of Territorial Identity, SAGE, London 1982. SHARPE L.J. : Decentralist Trends in Western Democracies, SAGE, London 1979.

3. Pour l'approche institutionnelle, voir par exemple : PUGET $H$. : Les Institutions Administratives Etrangères, Paris (Dalloz), 1969 ; ou MACRIDIS R.C. : The Study of Comparative Government, New York (Doubleday and Co) 1955 ; ou DUVERGER M. : Institutions politiques et Droit Constitutionnel, Paris (P.U.F. et Themis) 1975 (14ème édition).

4. Voir BLONDEL $J$. : An Introduction to Comparative Government, London (WEIDENFELD et NICOLSON) 1969 ; ou BLONDEL J., HERMAN V. : Workbook for Comparative Government, London (WEIDENFELD et NICOLSON) 1972. Et (toujours) WEBER M. : Economie et Société, Tome I, Paris (Plon) 1971. ARON R. : Democratie et Totalitarisme, Paris (N.R.F. Idées) 1965. FINER S.E. : Comparative Government, Harmondsworth, New York, Victoria, etc (Penguin books) 1970.

5. Voir pour l'Angleterre par exemple, la collection de BARRETT S., FUDGE C.: Policy and Action, Essays on the Implementation of Public Policy, London \& New York (Methuen) 1981 ; ou pour l'Allemagne de l'Ouest, les deux volumes rassemblant des travaux d'une unité de recherche financée par la Fondation Nationale de Recherche de l'Allemagne de l'Ouest, édités par R. MAYNTZ (Hrsg.) : Implementation Politischer Programme, Empirische Forschungsberichte, Königstein Ts. (Athenäum) 1980 ; ainsi que : Implementation Politischer Programme 11, Ansätze zur Theoriebildung, Opladen (Westdeutscher Verlag) 1983. La situation en France est décrite dans I'article de NIOCHE J.P. : De l'Evaluation à l'Analyse des Politiques Publiques, dans Revue Française de Science Politique XXXII (1982) 1, février 1982, pp. $32-61$.

Les notes $6,7,8$ et 9 renvoient à la bibliographie ; (recherches présentées lors du WZB Forum 1983). 


\section{BIBLIOGRAPHIE}

- ALMOND G.E. : A Functional Approach to Comparative Politics, in ALMOND G.E. et COLEMAN (eds) : The Politics of the Developing Areas, Princeton 1960.

- ALMOND G.E. et POWELL G.B. : Comparative Politics : A developmental Approach, Boston (Little, Brown \& Co), 1967.

- ASHFORD D. (ed) : Comparing Public Policies. New Concepts and Methods, Beverly Hill and London (SAGE), 1978.

- ASHFORD D. : The Structural Analysis of Policy or Institutions Really do Mater, in ASHFORD D. (ed) : Comparing Public Policies, 1978, p. 81-98.

- BARDACH E. : The Implementation Game : What Happens after a Bill Becomes a Law, Cambridge, Massachussetts, London, 1977.

- CAMPBELL D. : Reform as Experiments, in American Psychologist, $n^{\circ} 24$ (avril) 1969, p. 409.429.

- EASTON D. : A system Analysis of Political Life, New York (Wiley), 1965.

- heIdenheimer A., HeClo H., TEICH AdAMS C. : Comparative Public Policy. The Politics of Social Choice in Europe and America, (St Martin's Press Inc.), 1975.

- HUFNER K., MEYER J., NAUMANN J. : Comparative Education Policy Research - Putting Recent Trends into a World Society Perspective. Publication spéciale pour le WZB Forum 1983 "Cross National Policy Research», Berlin, 1983.

- LOWI T. : Four Systems of Policy, Politics and Choice, in Public Administration Review $n^{\circ} 32$ (July/August) pp. 298-310, 1972.

- LUEBBERT G., WILENSKY H., HAHN S., JAMIESON A. : Comparative Social Policy : Theories, Methods Findings. Publication spéciale pour le WZB Forum 1983, "Cross National Policy Research», Berlin, 1983.

- MERTON R.K. : Social Theory and Social Structure, édition révisée, New York (Free Press of Glencoe), 1957.

- MITCHELL W.R. : The American Policy, New York (The Free Press), 1962. 
Les politiques publiques comparées : tourisme intelligent ou vrai progrès ? 61 Le cas des politiques comparées de l'environnement

-PARSONS T. : The Social System, New York (Free Press of Glencoe), 1951.

- PARSONS T. : Structure and Process in Modern Societies, New York (Free Press of Glencoe), 1960.

PRESSMAN G.L., WILDAVSKI A.D. : Implementation : How Great Expectations in Washington are Dashed in Oakland, or Why it's Amazing that Federal Programs Work at all, Berkeley (University of California Press), 1973.

- STRUMPEL B., SCHOLZ J. : Comparative Studies of Economic Processes : A Litterature Review, Publication spéciale pour WZB Forum 1983, «Cross National Policy Research», Berlin, 1983.

- VOGEL D., KUN V. : The Comparative Study of Environmental Policy : A Review of Litterature. Publication spéciale pour le WZB Forum «Cross National Policy Research», Berlin, décembre 1983.

- WILDAVSKY A.D. : Doing More and Using Less. Utilization of Research as a Result of Regime, in publication spéciale pour le WZB Forum 1983, "Cross National Policy Research», Berlin, 1983. 
BIBLIOGRAPHIE CHOISIE PAR VOGEL, 1983, pp. 133-134

1. BRICKMAN R., JASANOFF S. and ILGEN T., Chemical Regulation and Cancer : A Cross-National Study of Policy and Politics, Cornell University, Ithaca, New York, 1982.

2. GUSMAN S., VON MOLTKe K., IRWIN F. and WHITEHEAD C., Public Policy for Chemicals, National and International Issues, The Conservation Foundation, Washington, D.C., 1980.

3. KELMAN, STEVEN, Regulating America, Regulation Sweden : A Comparative Study of Occupational Safety and Health Policy. MIT Press, Cambridge, Massachusetts, 1981.

4. KNOEPFEL P., Comparative Analysis of the Implementation of $\mathrm{SO}_{2}$ Air Quality Control Policies in Europe, Conceptual Framework and First Results, International Institute for Environment and Society, Berlin, 1981.

KNOEPFEL P. and WEIDNER H., F ormulation and Implementation of Air Quality Programs : Patterns of Interest Consideration ,Policy and Politics, Vol. 10, n 1 (1982), pp. 85-109.

KNOEPFEL P. and WEIDNER H., Implementing Air Quality Control Programs in Europe : Some Results of a Comparative Study, in Paul DOWNING and Kenneth HANF, International Comparisons in Implementing Pollution Laws. Boston : Lumer-Nijhoff Publishing, 1983.

KNOEPFEL P., WEIDNER H. and HANF K., International Comparative Analysis of Program Formation and Implementation in $\mathrm{SO}_{2}$ Air Pollution Control Policies in the ECE Countries and Switzerland : Analytical Framework and Research Guidelines for the National Research Teams, IIES, Science Center Berlin, June 1980.

KNOEPFEL P., WEIDNER $H$. and HANF K., The Implementation of $\mathrm{SO}_{2}$ Control Policy in the European Community and Switzerland, Air Pollution Control : National and International Perspectives, Aspen Institute Conference, April 8-11, 1979.

5. KUNREUTHER H. and LINNEROOTH J., Risk Analysis and Decision Processes : The Siting of LEG Facilities in Four Countries, IIASA, Laxenburg, Austria, March 1982. 
6. LUNDQVIST L., Do Political Structures Matter in Environmental Politics ? The Case of Air Pollution Control in Canada, Sweden, and the United States, Canadian Public Administration, Spring 1974.

7. LUNDQVIST L., The Hare and the Tortoise : Clean Air Policies in the United States and Sweden, University of Michigan Press, Ann Arbor, Michigan, 1980.

8. MANGUN, W. R., The Public Administration of Environmental Policy : A Comparative Analysis of the United States and West Germany, Institute of German Studies, Indiana University, Bloomington, Indiana, 1977.

9. NELKIN D. and POLLACK M., The Atom Besieged : Anti-Nuclear Movements in France and Germany, MIT Press, May 1980.

10. SOLESBURY W., Issues and Innovations in Environmental Policy in Britain, West Germany and California, Policy Analysis, Winter 1976.

11. VOGEL D., Coercion Versus Consultation : A comparison of Environmental Protection Policy in the United States and Great Britain, presented to the Annual Meeting of the American Political Science Association, Denver, Colorado, 1980.

VOGEL D., Cooperative Regulation : Environmental Protection in Great Britain, Public Interest, Summer 1983.

12. WANDESFORDE-SMITH G., Environmental Impact Assessment and the European Community, Zeitschift fur Umweltpolitik, 1979, pp. 35-76.

WANDESFORDE-SMITH G., Environmental Impact Assessment and the Politics of Development in Europe, in O'RIORDAN and TURNER, Progress in Resource Management and Environmental Planning, Vol. 2, 1980.

WANDESFORDE-SMITH G., Projects, Policies and Environmental Impact Assessment : A Look Inside California's Black Box, Science Center Berlin, 1978.

WANDESFORDE-SMITH G., Varieties of Environmental Impact Assessment : An International Analysis, Milieu en Recht, 1979, pp. 4-5. 